\title{
Establishment of Visual Supervision Mechanism for on - site Supervision of Electric Power Engineering
}

\author{
Zhang Yunshan, Liu Guofu, and Zhang Lei \\ Economic and Technological Research Institute, Liaoning Electric Power Co., Ltd. State Grid (Liaoning Electric Power \\ Construction Supervision Co., Ltd), Shenyang, China
}

\begin{abstract}
The quality of power engineering is an important guarantee for the power supply. The existing engineering supervision mainly relies on manual completion and lacks effective supervision mechanism. From the perspective of power engineering site supervision, the function and significance of visualization supervision mechanism are introduced, the application of visualization of power industry is enumerated, the necessary elements of visualization supervision of power engineering is summed up, and the visual supervision mechanism establishing for power engineering is discussed. The establishment of visualization regulatory mechanisms has a role in upgrading for power engineering site supervision and quality control.
\end{abstract}

\section{Introduction}

In order to ensure the quality and progress of the project, the supervisor should supervise and manage the construction progress, and directly determines the quality of the project. With the development of society and economy, the demand for electricity increased, the number of power supply enterprises in power engineering construction projects increased year by year. The supervision tasks corresponding increase in the power engineering. At present, the number of electric power projects in transmission and substation is relatively large, and the field is scattered in various cities. The management means are relatively simple, mainly through manual feedback on the situation, the information content mainly relies on the text, the photo records, and the provincial and the city company can not grasp the project in real time as well as the supervision situation. The effective supervision and control mechanism is lack for the project site supervision, and the supervision process problems can not be found. The visual data files are lack in power engineering supervision and management, and the query and trace technical means are more backward[1].

In the project site supervision work, the supervisors often record the situation on the spot, including supervision, rectification matters, problems and the next stage of work and so on. At present in the project site, the supervisor mainly relies on the manual record and the photograph way, records the project supervision key and the data[2]. Manual notes use text records; descript the scene supervision and problems. Photo recording methods mainly use digital cameras or mobile phones shoot construction site related images. The supervision staff can not fully show the scene supervision situation, such as the project quality, the progress of the problem and the lack of intuitive presentation, failed upload images and information in the field. The hidden dangers of electrical engineering safety and quality aspects are easily leaded. Therefore, for the on-site supervision of power engineering, the establishment of centralized visualization on-site supervision mechanism and realtime understanding of the project site situation are of great significance to strengthen the project supervision and control and improve the quality of the project[3].

\section{Power visualization applications}

Power industry visualization applications came out and developed with the visual technology, and on going construction and upgrading. Visualization technology applications are mainly used to solve the power of the professional security protection, equipment monitoring, real-time recording, service monitoring, remote command and other issues. Visual technology in the power industry site applications includes: substation monitoring, transmission line monitoring, and distribution network operation monitoring, emergency command, power supply business hall monitoring. Visualization application is realized by the video surveillance system; the real-time collection of video files will be timely transmission to the monitoring center, and real-time dynamic report the situation of monitoring point, find problems in times and process it[4].

The power professional visual construction and application are as follows[5].

\subsection{Substation security monitoring}

With unattended substation gradually increased, in order to ensure the safe operation of power grid and solve the 
problem of unattended substation security, power supply company has gradually implemented the substation remote video surveillance system. Substation video surveillance system is designed to achieve substation safety protection and operation of remote visual management. Improve the safety and reliability of substation operation and maintenance. Substation install a the camera in the indoor and outdoor, it used to complete image acquisition of the important areas, camera images collected to the station hard disk recorders, use streaming media service module the video stream to the request to connect the workstation through the LAN forwarding.

\subsection{Transmission line monitoring}

In the process of transmission line operation, the line conductor position, sag, ice and other running status are needed to regularly check. Transmission video image monitoring system is installed on the line, so that the transmission line is running in the visual monitoring, the scene surroundings are real-time monitored by digital video compression technology, low power technology, GPRS / CDMA / 3G / 4G wireless communication technology, solar power technology. Line managers can remotely view the line monitoring images or regularly upload image information, in order to achieve real-time monitoring of the transmission line all-weather.

\subsection{Distribution network operation visualization}

At present, the automation of the distribution network has realized the running data of the distribution line, the running status of the switching equipment and the related data, and transmitted to the background monitoring center through the optical fiber communication and GPRS communication. The background monitoring center can carry on the remote control operation to the remote equipment and tuning settings. Distribution automation increase "remote" on the basis of "four remote" to achieve remote real-time video surveillance distribution line switching equipment operation. "Remote" terminal video surveillance equipment using network high-definition camera, the camera itself directly to the video acquisition of analog signals into digital models, and directly transfer to the system background by the wireless router .

\subsection{Emergency Command Visualization}

Power dispatching, safety supervision and other professional in the field operations or troubleshooting need remote command site personnel view the fault node, and analyze the cause of the failure, quickly deal with field failure. Emergency command video system is installed in the vehicle, the camera is installed in the roof position; it can rotate 360 degrees and cover the scene panorama. The camera is connected to the in-car video server, and the video server transmits the video signal back to the dispatching command center through the wireless website. So that experts in the command center can view the job or accident scene to buy the number.

\subsection{Business hall video surveillance}

Business hall as a power supply office of the external service window, its quality of service on the power supply bureau has a direct impact on the image. In order to ensure the normal operation of the business hall and improve the quality of service power supply bureau must strengthen the management of the business hall. Complete real-time video on the basis of improving the quality of business services, to ensure the safe and orderly operation of the business hall. In the business hall window, counter and other locations, there are many real-time video surveillance management systems; all install a high-definition camera, real-time record of customer and customer service status, to ensure to archive the whole process of customer service video.

\section{Power engineering visualization elements}

Through the power industry visualization application and development power engineering visualization are mostly fixed place application, camera fixed shooting and easy to move after installation can be seen in the substation, transmission, distribution, marketing and other professionals, at the same time the scene has a stable power supply and network supply, the equipment supply and communication transmission problems are not regarded. This makes the difficulty of visual construction and cost greatly reduced. Camera equipments are installed in the indoor and outdoor areas, there are no restrictions on space and requirements, and so in the visual system to build the process does not need to consider the size of the camera equipment. There are a variety of camera types, including gun-type cameras and dome cameras and infrared cameras.

Table 1. Power visualization monitoring system content

\begin{tabular}{|c|c|c|c|}
\hline $\begin{array}{c}\text { Application } \\
\text { Environment }\end{array}$ & Camera type & Network & $\begin{array}{c}\text { Intelligent } \\
\text { identification }\end{array}$ \\
\hline Substation & $\begin{array}{c}\text { Simulation, } \\
\text { high definition }\end{array}$ & Wired LAN & Yes \\
\hline Transmission line & $\begin{array}{c}\text { Simulation, } \\
\text { high definition }\end{array}$ & Wired LAN & No \\
\hline Distribution lines & $\begin{array}{c}\text { Simulation, } \\
\text { high definition }\end{array}$ & Wired LAN & No \\
\hline $\begin{array}{c}\text { Emergency } \\
\text { command }\end{array}$ & high definition & $\begin{array}{c}\text { Wireless } \\
\text { LAN }\end{array}$ & No \\
\hline $\begin{array}{c}\text { Business hall } \\
\text { monitoring }\end{array}$ & $\begin{array}{c}\text { Simulation, } \\
\text { high definition }\end{array}$ & Wired LAN & No \\
\hline
\end{tabular}

Outdoor monitoring commonly used standard definition camera, resolution 720P, indoor high-definition camera, resolution up to $1080 \mathrm{P}$. In the substation, transmission line visual monitoring has increased the intelligent behavior analysis function, through the video image recognition, the substation, the line operation abnormal situation, carries on the automatic recognition discrimination. The power visualization monitoring system building content is shown in Table 1.

There is a clear difference between the power engineering site and power substation, transmission, distribution, safety supervision, marketing and other 
business. Power engineering site are under construction in the substation project and transmission line project, and their construction period are always several years. The visualization systems and equipment are only used during construction and will no longer be used on site after completion of the project for the power engineering sites. While other professional visualization systems will be running on-site after the completion of the project. Before the construction of the electric power is completed, the temporary power or generators is used to provide power for the project, therefore a stable power supply can not be guaranteed. Most power engineering sites do not provide power supply for visualization systems and equipment. As the site construction site and the environment often change, the installation of camera equipment can not be in the ideal site. The lack of fullangle and real-time monitoring mechanism can not guarantee the construction of power engineering supervision in place and can not be intuitive and accurate response to the project site happening.

There is no communication equipment at the power engineering site, can not provide a fixed network communication environment. In the video transmission of the camera, the use of traditional wired connection, can only be used as temporary during the project; line laying costs are high, and the construction period is long, the construction process and after completion will be removed. Construction site environment and other physical factors also increased the difficulty of erection of cables. Project site camera head repeatedly disassembling likely cause damage to the camera, affect the camera life, and the camera is larger and lack of flexibility to increase the supervision and construction workers burden.

In the power engineering construction site supervision work, the construction progress and the scene situation are needed to check by going to the various construction sites, once the supervision is not timely, supervision lack of important basis and information is caused easily. Even if the visualization system be used in the project site, only a single point of management is existed, the lack of centralized unified monitoring and management reduce the efficiency of the visualization system to increase the cost of supervision and management work.

In order to realize the establishment and application of visualization supervision of power engineering, it is necessary to solve the problems such as camera fixed, power supply stability and communication network. The key elements of visualization supervision of power engineering site supervision are as follows.

- Convenient for supervisors to carry and use on site.

- Small size, easy to move, easy to operate.

- Be used for a long time without power.

- With wireless communication and data transmission function.

- A number of project visual centralized management.

\section{Power scene visualization establishment methods}

Power engineering construction involves transmission, substation and other engineering sites, is affected by the environmental conditions of the project site, resulting in more difficult visualization supervision, and thus the video-related device equipment can not be installed. Therefore, the visualization and mobility of the visualization device and equipment is the key to realize the visual supervision of power engineering site supervision. In the field of electric power engineering, the comprehensive utilization of portable, mobile and fixed visualization devices, they have high-definition image acquisition, video storage, voice recording and transmission and communication functions, can real-time record the project supervision progress and record fullangle and multi-dimensional situation. Visualization device has good portability and mobility, is suitable for all kinds of power engineering site supervision applications, can complete the scene image, video recording, collection, transmission, and is easy to carry and use the supervision staff, do not increase the on-site staff burden, and deploy and install at the supervision site quickly.

The wearable visual collection device due to the use of embedded design, it can be easily installed on the helmet, shoulders, chest, easy to carry and use the officers. The wearable visual collection device integrates highdefinition video capture, image processing, video storage, voice recording, communication function, complete project supervision site visualization records; supports wireless communication and storage backup mode, completes on-site supervision visualization upload. To ensure that the wearing device can work longer than 12 hours the large capacity lithium battery is used. The onsite use of mobile acquisition devices are suitable and easy to carry. Built-in high-definition camera, highdefinition camera ball machine, storage devices, communication devices, fixed bracket, it can quickly be installed in the supervision of the site. According to the supervision of the scene situation, the mobile visualization device can use the fixed bracket, be placed in the field of any location, and real-time collect allweather supervision site work content and circumstances, and assist the crew to record the scene to complete the supervision of the scene image and video recording. Both active and passive power supply for visualization equipment is to meet a variety of engineering site environment applications. The use of solar panels and batteries can complete the visualization of the device under the passive environment of the power supply. The visual acquisition device with active environment and passive environment can all-weather real-time collect the on-site supervision work of the situation.

The difficulty of on-site monitoring is increasing because power engineering site coverage is relatively wide and the project time is long. In order to ensure the supervision and control of the power engineering site supervision business, it is necessary to realize the whole process monitoring of the project site. The full coverage visualization device application is the basis and guarantee of power engineering site supervision. In the power engineering site, the power engineering supervision on-site visualization can be achieved by the 
use of wireless or wired communication to support portable visualization devices, mobile visualization devices or fixed visualization device access applications, covering the power engineering supervision site and supervision of the various processes. In the project supervision site, the application of wireless transmission technology and wireless communication network, the formation of transmission network connection and completing the supervision of the scene multi-channel visual video wireless collection and finishing, all-round displaying the supervision site can meet all kinds needs of engineering site environment applications. In the power engineering supervision site, the use of $4 \mathrm{G}$ communication network, the establishment of wireless transmission network and wireless hotspot AP, and visualization system platform can establish a network connection and upload supervision site visualization images. Because of the strong scalability, the visualization regulatory mechanisms can be flexibly and easily installed and deployed to provide high reliability and ensure uninterrupted visual monitoring.

Power engineering site visual supervision needs to rely on the system platform to real-time check the view of the project supervision site and the process of retrospective project records. The centralized management of visualization process management has been achieved after power engineering site supervision visualization resources collection and uploaded to the unified system platform. It can monitor the scene of the various power engineering supervision of the unified data management, focus on the various supervision of the scene, the progress of the project, the construction of the management staff to master the work of supervision work, sum up project supervision process visualization files; real-time check the supervision site visualization of the image, achieve on-site visual remote call, command and coordinate on-site supervisors work; establish the project supervision visualization process archives, in the process of project supervise and display project site supervision of image and video data, enrich the project supervision process visual query trace function, check playback power engineering supervision live situation.

\section{Conclusions}

In view of the problems and requirements of on-site supervision of electric power engineering, the application of visualization technology is innovated, and a visual supervisory mechanism is established at the power engineering site. The establishment of visualization regulatory mechanisms can focus on the management of power engineering site, achieve full supervision of power engineering site supervision, and be convenient and quick complete supervision on-site inspection and supervision; and can improve the efficiency of the scene, reduce the strength of the supervision staff, timely detect the problems in the construction process, enrich the site supervision and control technology to further enhance the quality and level of power engineering site management.

\section{References}

1. XIAO Donghui, LIN Li. Solution Scheme of Integrated Video Surveillance Platform of Power System[J]. Automation of Electric Power Systems, 2013, 5: 74-80.

2. XIAO Xing-quan, LI Fu-xiang. Discussion on the Interconnection of Sichuan Power Video Monitoring System Platforms[J]. Telecommunications for Electric Power System, 2010, 7: 30-35.

3. CUI Yanming, LIU Xiaoxian, MA Chao, CHEN Jianning. Standards of Grid Video Surveillance System and Interface Technology[J]. Automation of Electric Power Systems, 2010, 20: 13-16.

4. ZHANG Jinzhi. Real-time Video Monitoring and Management System of Power Supply Business Hall Based on Local Area Network Transmission[J]. Guangdong Electric Power, 2009, 2: 58-62.

5. CHEN Xinhe, SONG Wei, LU Ying. Information Integration Demand Analysis and Data Modeling for Interactive Electric Power Business Hall[J]. Automation of Electric Power Systems, 2013, 7: 7781. 